\title{
Updated guidelines on screening for gestational diabetes
}

\author{
This article was published in the following Dove Press journal: \\ International Journal of Women's Health \\ 19 May 2015 \\ Number of times this article has been viewed
}

\author{
Yashdeep Gupta' \\ Bharti Kalra ${ }^{2}$ \\ Manash P Baruah ${ }^{3}$ \\ Rajiv Singla ${ }^{4}$ \\ Sanjay Kalra ${ }^{2}$ \\ 'Department of Medicine, \\ Government Medical College and \\ Hospital, Chandigarh, India; ${ }^{2}$ Bharti \\ Hospital, Karnal, Haryana, India; ${ }^{3}$ Excel \\ Center, Guwahati, Assam, India; ${ }^{4}$ Saket \\ City Hospital, New Delhi, India
}

Correspondence: Yashdeep Gupta Department of Medicine, Government Medical College and Hospital, Sector 32, Chandigarh 160030, India

Tel +9l 964612 I573

Email yash_deep_gupta@yahoo.co.in

\begin{abstract}
Gestational diabetes mellitus (GDM) is associated with an increased risk of complications for both mother and baby during pregnancy as well as in the postpartum period. Screening and identifying these high-risk women is important to improve short- and long-term maternal and fetal outcomes. However, there is a lack of international uniformity in the approach to the screening and diagnosis of GDM. The main purpose of this review is to provide an update on screening for GDM and overt diabetes during pregnancy, and discuss the controversies in this field. We take on debatable issues such as adoption of the new International association of diabetes and pregnancy study groups criteria instead of the Carpenter and Coustan criteria, one-step versus two-step screening, universal screening versus high-risk screening before 24 weeks of gestation for overt diabetes, and, finally, the role of $\mathrm{HbA}_{1 \mathrm{c}}$ as a screening test of GDM. This discussion is followed by a review of recommendations by professional bodies. Certain clinical situations, in which a pragmatic approach is needed, are highlighted to provide a comprehensive overview of the subject.
\end{abstract}

Keywords: pregnancy, guidelines, IADPSG, GDM, Carpenter and Coustan criteria

\section{Introduction}

Gestational diabetes mellitus (GDM) has classically been defined as any glucose intolerance first identified during pregnancy. ${ }^{1}$ Recently, the American Diabetes Association (ADA) defined it as "Diabetes diagnosed in the second or third trimester of pregnancy that is not clearly overt diabetes". ${ }^{2}$ However, as per IADPSG (International association of diabetes and pregnancy study groups) criteria, women can be diagnosed to have GDM even in the first trimester, if fasting plasma glucose (FPG) is $\geq 5.1 \mathrm{mmol} / \mathrm{L}(92 \mathrm{mg} / \mathrm{dL})$, but $<7 \mathrm{mmol} / \mathrm{L}(126 \mathrm{mg} / \mathrm{dL}){ }^{3}$

GDM is associated with an increased risk of complications for both mother and baby, during pregnancy as well as in the postpartum period. Screening and identifying these high-risk women is important to improve short and long-term maternal and fetal outcomes. ${ }^{4}$ However, there is lack of international uniformity in the approach to the screening and diagnosis of GDM. ${ }^{5}$ This is surprising, given that the strategies for making a diagnosis of diabetes mellitus are uniform across the world. ${ }^{2}$ The main reason for the diagnostic dilemma of GDM is the large number of procedures and glucose cutoffs proposed for the diagnosis of glucose intolerance in pregnancy. ${ }^{6}$ The first diagnostic criteria proposed by O'Sullivan in 1964 and its subsequent modifications (Carpenter and Coustan) were based on the maternal risk of developing type 2 diabetes, rather than on pregnancy outcomes. Recently, the recommendations from IADPSG attempt to redefine GDM in terms of adverse pregnancy outcomes, based on Hyperglycemia and Adverse Pregnancy Outcome (HAPO) study results. ${ }^{6}$ But, we are still far from attaining a holistic criteria which is based on both short and long-term outcomes. 
The main purpose of this review is to provide an update on screening for GDM and overt diabetes during pregnancy, and discuss the controversies in this field. We take on debatable issues such as adoption of the new IADPSG criteria instead of the Carpenter and Coustan criteria, one-step versus two-step screening, universal screening versus high-risk screening before 24 weeks of gestation for overt diabetes, and, finally, the role of $\mathrm{HbA}_{1 \mathrm{c}}$ as a screening test of GDM. This discussion is followed by a review of recommendations by professional bodies. Certain clinical situations, in which a pragmatic approach is needed, are highlighted to provide a comprehensive overview of the subject.

\section{Evolution of screening criteria for GDM}

The first systematic evaluation of the oral glucose tolerance test (OGTT) for the diagnosis of diabetes in pregnancy was done by O'Sullivan et al in the 1950s and 1960s. Since then, the 100-g OGTT has undergone extensive modifications, to its present form, that is, Carpenter and Coustan criteria (1982). ${ }^{6,7}$ Recently, IADPSG criteria have also come into the picture. ${ }^{3}$

\section{I00-g OGTT}

The original diagnostic criteria, based on blood glucose testing before and hourly for 3 hours after 100-g glucose intake for GDM, were proposed by O'Sullivan and Mahan in $1964,{ }^{8}$ based on a series of 752 women who underwent OGTTs during pregnancy. Means and standard deviations (SDs) were derived for each of four whole blood glucose values (defined as mean plus 1, 2, or $3 \mathrm{SD}$ ). O'Sullivan and Mahan decided that two abnormal values would be needed for GDM diagnosis. The decision was based upon the desire to avoid misclassification due to laboratory error or the occasional individual with a single high glucose peak due to rapid absorption. Approximately $2 \%$ of pregnant women fulfilled the criteria of mean plus $2 \mathrm{SD}$, and these criteria became the basis for the diagnosis of GDM in the USA. The diagnostic criteria were applied to a separate group of 1,013 women who underwent 100 -g OGTTs during their pregnancy. The risk of subsequent diabetes was $27 \%$ after a follow-up period of 8 years, when values at $2 \mathrm{SD}$ were used as the diagnostic threshold during pregnancy. ${ }^{6-8}$

In 1979, the National Diabetes Data Group criteria (NDDG) converted the whole blood glucose thresholds to the plasma values (approximately $14 \%$ higher as compared with the original O'Sullivan and Mahan criteria), in response to the general change in laboratory standards from whole blood to plasma or serum. ${ }^{6,9}$ Subsequently, new laboratory technology for glucose measurements using glucose oxidase and hexokinase methods, led to the formulation of the Carpenter and Coustan criteria. ${ }^{6,10}$ The original O'Sullivan and Mahan criteria were established using Somogyi-Nelson technology. The Somogyi-Nelson method is not specific for glucose, and also measures approximately $0.27 \mathrm{mmol} / \mathrm{L}(5 \mathrm{mg} / \mathrm{dL})$ of nonglucose reducing substances. Glucose oxidase and hexokinase methods, on the other hand, measure only glucose. In 1982, Carpenter and Coustan used the glucose oxidase method to derive a set of criteria by first subtracting $5 \mathrm{mg} / \mathrm{dL}$ from O'Sullivan and Mahan's original values and then adding 14\% to each (to account for the conversion from whole blood to plasma glucose values), and, finally, rounding to the nearest $5 \mathrm{mg} / \mathrm{dL}(0.27 \mathrm{mmol} / \mathrm{L})$. This formulated the Carpenter and Coustan criteria. . $^{6,710}$

\section{5-g OGTT}

The initial recommendation for using 75-g OGTT in pregnancy was from the World Health Organization (WHO). The WHO used the same criteria for diagnosing diabetes both during and outside of pregnancy. ${ }^{11}$ This approach was criticized, as it ignored the physiological changes in carbohydrate metabolism that occurs during pregnancy. In 1999, the $\mathrm{WHO}^{11}$ lowered the threshold for FPG from $7.8 \mathrm{mmol} / \mathrm{L}$ $(140 \mathrm{mg} / \mathrm{dL})$ to $7.0 \mathrm{mmol} / \mathrm{L}(126 \mathrm{mg} / \mathrm{dL})$ and recommended that pregnant women meeting the criteria for diabetes mellitus or impaired glucose tolerance (IGT) be classified as having GDM.

All these criteria had a common problem, namely, they were validated for predicting the future risk of diabetes only in the mother. ${ }^{6-10}$ A need was felt to derive new criteria linking level of glycemia to pregnancy and fetal outcomes. The current 75-g IADPSG criteria have been devised keeping this fact in mind and evaluating evidence that associates abnormal glucose tolerance in pregnancy with adverse perinatal outcomes. ${ }^{3}$ Unblinded studies since 1995 have shown adverse perinatal outcomes to be linearly linked with glycemic levels in gestation. ${ }^{5}$ The landmark study in this respect was the HAPO study. ${ }^{12}$ The HAPO study was a large, multicenter, multinational, epidemiologic study in which 23,316 women (>30 times larger than the O'Sullivan cohort) underwent blinded 2-hour, three-sample, 75-g OGTTs at 24-32 weeks of gestation. All women with a fasting plasma glucose $(\mathrm{FPG}) \leq 5.8 \mathrm{mmol} / \mathrm{L}(105 \mathrm{mg} / \mathrm{dL})$ and 2 hours values up to $11.1 \mathrm{mmol} / \mathrm{L}(200 \mathrm{mg} / \mathrm{dL})$ were included. The HAPO clearly established a linear relationship between each of the glucose values (fasting, 1 hour, and 2 hours) on OGTT and a 
broad range of predefined pregnancy outcomes. The primary outcomes in the HAPO study were the frequency of large-forgestational-age (LGA, >90th centile) babies, primary cesarean section, clinical neonatal hypoglycemia, and neonatal hyperinsulinemia. All of these primary outcomes as well as secondary outcomes like fetal adiposity, preeclampsia, and birth trauma/shoulder dystocia were related to each of the maternal OGTT glucose results in a continuous fashion. The independent associations of hyperglycemia with pregnancy outcomes persisted after extensive adjustment for potential confounders including maternal body mass index (BMI), age, height, mean arterial pressure, and parity. ${ }^{5,12}$

In 2008, the IADPSG recommended the establishment of new diagnostic criteria for GDM based on data from the HAPO study. The diagnostic threshold would have to be somewhat arbitrary, because of the linear association between glycemic values and pregnancy outcomes. The diagnostic thresholds that were decided by the IADPSG consensus panel are based on glucose levels that give $75 \%$ increased risk of birth weight $\geq 90$ th centile, cord C peptide $\geq 90$ th centile, and percentage body fat $\geq 90$ th centile as compared with mean glucose levels of women of HAPO cohort. On this basis, the IADPSG recommended a fasting glucose level of $5.1 \mathrm{mmol} / \mathrm{L}$ (92 mg/dL), a 1-hour level of $10.0 \mathrm{mmol} / \mathrm{L}$ (180 mg/dL), or a 2 -hour value of $8.5 \mathrm{mmol} / \mathrm{L}(153 \mathrm{mg} / \mathrm{dL})$ as diagnostic for GDM. ${ }^{3}$ The IADPSG criteria have been endorsed by WHO, ADA, and the Endocrine Society of USA. 2,13,14

\section{Controversy: should IADPSG criteria be adopted universally?}

The prevalence of GDM increases by two- to threefold if the IADPSG criterion is adopted for screening. There is an ongoing debate whether such an increase in prevalence allows identification of previously ignored risks, or results in overmedicalization of healthy pregnancies. ${ }^{5,15-20}$ There is a need to closely look at arguments on both sides.

\section{Arguments against adopting IADPSG criteria universally \\ Weak association of complications with glycemic levels}

The lack of association between perinatal mortality and higher blood glucose levels, as observed in the HAPO study, has been used as a strong counterpoint by the detractors of the IADPSG criteria. ${ }^{12,16}$ The association between secondary endpoints (shoulder dystocia, premature delivery, hyperbilirubinemia, and preeclampsia) and glycemic levels was significant, but clinical relevance of this finding is limited as these complications are relatively infrequent. The association between maternal blood glucose and certain endpoints like neonatal hypoglycemia, birth weight, cesarean section rate, and preeclampsia either disappeared or became weak when maternal characteristics such as BMI were taken into account. ${ }^{12,16-19}$

\section{Increased rate of intervention and adverse effects}

The diagnosis of GDM, irrespective of the degree of glucose control achieved, per se leads to increased interventions, earlier delivery, an increased cesarean section rate (even if birth weight is normalized by treatment), and a higher number of neonatal admissions to special care nurseries. ${ }^{16,17,20}$ This phenomenon has been observed in the Australian Carbohydrate Intolerance Study in Pregnant Women (ACHOIS). ${ }^{21}$ The basis for this finding is not entirely clear, but it may be related to the overcautious approach adopted by caregivers in pregnancies with diabetes. These complications may be more frequent in routine clinical settings, outside of controlled research settings. ${ }^{16,17}$

Lower diagnostic values will apparently lower target glucose values implying that more women will be treated with glucose-lowering therapy. This may increase the risk of maternal hypoglycemia and poor fetal growth, which in turn will contribute to metabolic disorders in adulthood. All these interventions create stress and anxiety among women diagnosed as having GDM. ${ }^{16,20}$

\section{Evidence of no treatment benefit for mild GDM from RCTs}

Two landmark randomized trials performed by Crowther et al (ACHOIS) and Landon et al (Maternal-Fetal Medicine Units, MFMU) influenced the recommendations for GDM diagnosis, by IADPSG. ${ }^{21,22}$ Analysts point out that these two trials were different from the HAPO study and should not have been considered along with it. ${ }^{16,17}$ The broad differences between them and the HAPO study are discussed here.

1. Trial design: These were intervention trials to investigate the effect of GDM treatment (diet and/or insulin) on perinatal outcomes, whereas the HAPO study was an observational study.

2. Inclusion/exclusion criteria: Unlike HAPO, where women irrespective of previous GDM were included, both studies excluded women with such a history.

3. Diagnostic criteria: Both studies used a two-step diagnostic procedure but with different diagnostic criteria. The criteria for the ACHOIS study were positive $50 \mathrm{~g}$ glucose challenge test (GCT) at 24-34 weeks' gestation 
$(\geq 7.8 \mathrm{mmol} / \mathrm{L}$ ) and $\mathrm{FPG} \leq 7.8 \mathrm{mmol} / \mathrm{L}$ and 2 hours value 7.8-11 mmol/L on 75-g OGTT. For the MFMU study, the criteria were positive $50 \mathrm{~g}$ GCT at 24-31 weeks' gestation $(7.5-11.1 \mathrm{mmol} / \mathrm{L})$ and $\mathrm{FPG} \leq 5.3 \mathrm{mmol} / \mathrm{L}$ and 2 or 3 postload levels elevated ( 1 hour $>10.0 ; 2$ hours $>8.6$; 3 hours $>7.8 \mathrm{mmol} / \mathrm{L}$ ) on 100 -g OGTT. The MFMU study excluded women with $\mathrm{FBG}$ values $>5.3 \mathrm{mmol} / \mathrm{L}$ (95 mg/dL), so the participants were less hyperglycemic than those of the ACHOIS cohort. The HAPO study used a single-step testing protocol without a prior GCT.

4. Outcomes: Both studies included perinatal mortality as a serious outcome, unlike in the HAPO study, in which perinatal mortality was not a part of the assessment. In the ACHOIS study, ${ }^{21}$ the routine care group showed a higher prevalence ( $4 \%$ vs $1 \%$ ) of composite primary outcome of "any serious perinatal complication." However, some of the deaths could not plausibly be attributed to the nontreatment of GDM: for example, one infant had a lethal congenital anomaly, and another had severe intrauterine growth retardation. In the MFMU trial, ${ }^{22}$ no difference was found between the groups in the composite primary outcome score.

\section{Meta-analysis of treatment trials}

A recent meta-analysis of the treatment RCTs (including, and dominated by, the ACHOIS and MFMU trials) concluded that the only pregnancy outcomes affected by lowering the threshold for detection and treatment of GDM are a reduction in macrosomia (odd ratio [OR] 0.38; 95\% CI, 0.30-0.49), LGA infants (OR 0.48; 95\% CI, 0.38-0.62), and the incidence of shoulder dystocia (OR $0.40 ; 95 \%$ CI, 0.21-0.75). ${ }^{23}$ In summary, the intervention trials in GDM confirmed that treatment contributes to a $2 \%-3 \%$ reduction in birth weight, leading to a lower incidence of "big babies" and shoulder dystocia. Moreover, the risk-benefit ratio for treatment is debatable. The authors of the ACHOIS trial calculated an NNT (number-needed-to-treat) of 34 to prevent "any serious perinatal complication," but an NNH (number-needed-to harm) of 11 for induction of labor or admission to neonatal nursery. ${ }^{21}$

\section{Arguments in favor of adopting IADPSG criteria universally}

Experts in favor of IADPSG criteria argue that the National Institute of Health (NIH) panel's report and other critiques have failed to address the issue of increasing prevalence of prediabetes and undiagnosed type 2 diabetes, outside pregnancy, in women of childbearing age. ${ }^{16-18,24}$ Moreover, considering the metabolic milieu in pregnancy, the threshold for diagnosis of diabetes has to be lower and more stringent than for the general population. Given the NHANES prevalence estimates for impaired glucose metabolism reaching $30 \%$ in women of childbearing age, the two- to threefold increase in prevalence of GDM, using the IADPSG approach may actually represent the true picture. $^{24}$

\section{Association of complications with glycemic levels}

According to the investigators of the HAPO study, glycemia was more strongly associated with the primary study outcomes as compared with maternal BMI..$^{25,26}$ The effects of these two variables on primary outcomes were additive, without a statistically significant interaction. ${ }^{25}$ The consensus panel chose features of diabetic fetopathy, namely, the frequency of birth weight $>90$ th centile, percent body fat $>90$ th centile, and cord C peptide $>90$ th centile for the determination of diagnostic glucose thresholds. These are key phenotypic features of babies directly related to the pathophysiology of GDM and may also link with future metabolic abnormalities in adulthood. The selected thresholds also identify an increased risk of more severe, though less frequent, adverse pregnancy outcomes. ${ }^{27}$

\section{Increase in adverse effects and interventions}

IADPSG recommendations represent a well-reasoned consensus view as to the levels of glycemia that are "sufficient to merit identification and treatment." These criteria, if thoughtfully implemented, will appropriately identify and allow treatment of the metabolic abnormalities of GDM. This will lead to well-established benefits, in terms of immediate pregnancy outcomes, and likely benefits, in terms of future maternal and offspring health. ${ }^{5,15}$

\section{Evidence of treatment benefit for mild GDM from RCTs}

Experts favoring the IADPSG criteria argue that there is a high degree of congruence in the results of these two trials (ACHOIS and MFMU), ${ }^{21,22}$ as discussed in detail here:

1. Glycemic threshold at entry: Despite different diagnostic criteria of the two trials, as compared with the HAPO study, the subjects recruited in all studies had glycemic values comparable to those in the HAPO study. 5,12,15,21,22 In the ACHOIS study, the FPG value was used only to exclude women, initially using FPG $7.8 \mathrm{mmol} / \mathrm{L}$ $(140 \mathrm{mg} / \mathrm{dL})$ and changing it later to $7.0 \mathrm{mmol} / \mathrm{L}$ $(126 \mathrm{mg} / \mathrm{dL})$ after a change in the WHO recommendations. Thus, it would have been possible for women with 
marked fasting hyperglycemia to be included in this trial. However, as this degree of fasting hyperglycemia is very uncommon in pregnancy when the 2-hour plasma glucose is $<11.1 \mathrm{mmol} / \mathrm{L}(200 \mathrm{mg} / \mathrm{dL})$, this criterion was rarely invoked. ${ }^{15,21}$ Landon et $\mathrm{al}^{22}$ applied a much more stringent criterion, excluding women with FPG $5.3 \mathrm{mmol} / \mathrm{L}$ (95 mg/dL).

The mean FPG of $4.8 \mathrm{mmol} / \mathrm{L}(86 \mathrm{mg} / \mathrm{dL})$ is similar in both ACHOIS and MFMU RCTs. The median 2-hour glucose of the ACHOIS cohort was actually close to the IADPSG recommended 2-hour diagnostic threshold, meaning that if this value alone were to be used for diagnosis, around $50 \%$ of these women, who benefited from intervention, would not have been diagnosed as having GDM. ${ }^{15}$

2. Advantages of treatment of GDM: Both studies showed a reduction in complications with the identification and active treatment of mild GDM. This was seen in terms of mean birth weight, frequency of LGA, ${ }^{21,22}$ reduction in fat mass, and in the reduction of shoulder dystocia. ${ }^{22}$ Hypertensive disorders of pregnancy (gestational hypertension and preeclampsia) were also substantially reduced by active GDM treatment. ${ }^{21,22}$ Maternal weight gain was lessened by active therapy. ${ }^{21,22}$ Induction of labor was increased by active treatment in the Crowther study, ${ }^{21}$ but not in the Landon study. ${ }^{22}$ The frequency of cesarean sections was unchanged in the ACHOIS study, ${ }^{21}$ and reduced in the MFMU study. ${ }^{22}$ A subsequent systematic review concluded that the observed reductions in LGA, shoulder dystocia, and preeclampsia are consistent across these and other available reports. ${ }^{28}$ As for perinatal mortality, the HAPO study was not powered to detect a difference in perinatal mortality, and for ethical reasons, the protocol was designed to minimize the risk of perinatal mortality.

\section{Consensus}

The relationship between hyperglycemia and adverse pregnancy outcomes is well defined through a large volume of congruent observational epidemiologic data, which were also considered for IADPSG recommendations.

The major observational studies have generally been performed with a 75-g OGTT, without prior GCT. The results, in particular the associations with excess fetal growth and its complications and the risk of pregnancy hypertension, remain consistent despite varying methods of analysis. No study found a clear diagnostic threshold or "inflection point" for any glucose measure above which risk increased sharply. Therefore, any decision regarding threshold values for GDM diagnosis will, by definition, be arbitrary, based on an individual or consensus view of the level of risk that is "sufficient" to merit the GDM label. The underlying principles of the IADPSG consensus process were 1) that women with equivalent levels of glucose-associated risk should be classified in a similar manner, and 2) that glucose criteria should be standardized internationally. ${ }^{27}$

\section{Universal testing for hyperglycemia in pregnancy in first trimester}

Pregnant women with overt diabetes and suboptimal blood glucose control in early pregnancy are at increased risk of having a fetus with congenital anomalies and are also at increased personal risk of worsening of diabetic retinopathy and nephropathy. Early diagnosis of previously undiscovered overt diabetes in a pregnant woman may allow for the rapid institution of therapy to mitigate these risks. As the frequency of obesity and T2DM in young adults is increasing worldwide, most guidelines now recommend screening for overt diabetes at the first prenatal visit, especially in high-risk groups. ${ }^{2,13,14,18,19,29}$

The cutoffs for tests, recommended to detect diabetes in early pregnancy are FPG: $126 \mathrm{mg} / \mathrm{dL}$ (7.0 mmol/L); random plasma glucose: $200 \mathrm{mg} / \mathrm{dL}(11.1 \mathrm{mmol} / \mathrm{L})$; or $\mathrm{HbA}_{1 \mathrm{c}}: 6.5 \%$ (47 mmol/mol), same as recommended for nonpregnant population. ${ }^{3}$ However, the WHO does not consider $\mathrm{HbA}_{1 \mathrm{c}}$ for the diagnosis of overt diabetes in pregnancy. ${ }^{14}$ It is generally considered that there is not enough evidence to recommend the screening and treatment of GDM before 24 weeks of gestation, ${ }^{30}$ and some professional organizations still recommend risk factor-based screening in the first trimester. ${ }^{18,19}$

IADPSG recommends that an FPG $92 \mathrm{mg} / \mathrm{dL}$ (5.1 mmol/L) in early pregnancy be classified as GDM. ${ }^{3}$ This recommendation remains highly debatable as the figure is based on data extrapolated from the cutoff value used on the 75-g OGTT later in pregnancy. A recent evaluation of the FPG in the first prenatal visit to diagnose GDM in People's Republic of China showed that an FPG level between 110 and $125 \mathrm{mg} / \mathrm{dL}$ (6.1-6.9 $\mathrm{mmol} / \mathrm{L})$ was a much better predictor of the development of GDM and that, for their population, an FPG level of $92 \mathrm{mg} / \mathrm{dL}(5.1 \mathrm{mmol} / \mathrm{L})$ at the first prenatal visit could not be supported as the criterion for the diagnosis of GDM. ${ }^{31}$

However, the recommendation of IADPSG for the diagnosis of overt diabetes is agreed upon even by the strong critics of the proposed criterion of IADPSG for the diagnosis of GDM. ${ }^{16}$ Based on available evidence, universal screening for GDM has to be carried out after 24 weeks, without any 
doubt or controversy. ${ }^{30}$ On the pragmatic side, it is worthwhile to test blood glucose in the first trimester to rule out overt diabetes. ${ }^{13}$

\section{Two-step screening: GCT followed by OGTT}

In the two-step screening approach, a 50-g GCT is followed by a 100-g, 3-hour OGTT if warranted by the results from the GCT. The screening threshold for GDM in the United States of either $7.8 \mathrm{mmol} / \mathrm{L}(140 \mathrm{mg} / \mathrm{dL})$ or $7.2 \mathrm{mmol} / \mathrm{L}$ (130 mg/dL) on the 50-g, 1-hour oral GCT is used. Those who screen positive are followed up by an oral 100-g glucose tolerance test (OGTT, in which four blood samples are drawn over a 3-hour period after a woman drinks $100 \mathrm{~g}$ of glucose solution). GDM is officially diagnosed with a positive result on two abnormal values on OGTT. The current diagnostic thresholds for the OGTT are: $\geq 5.3 \mathrm{mmol} / \mathrm{L}(95 \mathrm{mg} / \mathrm{dL})$ (fasting); $\geq 10 \mathrm{mmol} / \mathrm{L}(180 \mathrm{mg} / \mathrm{dL})$ (1 hour); $\geq 8.6 \mathrm{mmol} / \mathrm{L}$ (155 mg/dL) (2 hours); and $\geq 7.8 \mathrm{mmol} / \mathrm{L}(140 \mathrm{mg} / \mathrm{dL})$ (3 hours). In the USA, the American College of Obstetricians and Gynecologists (ACOG) along with the NIH still promotes a two-step process with initial 50-g nonfasting GCT. ${ }^{2}$

\section{Arguments favoring two-step method}

An advantage of the widely used two-step test (nonfasting $50 \mathrm{~g}$ glucose challenge, followed, if positive, by a fasting OGTT) is that women have to "fail" two tests to be diagnosed with GDM, so one can be more confident that indeed they have significant glucose intolerance. The proposed one-step test, while administratively more convenient, loses this discriminating power. ${ }^{16}$ This concern has been raised in view of poor reproducibility of OGTT. As illustrated by studies that have looked at the results of women having two 100-g OGTTs at short intervals (with two elevated glucose results out of four required to diagnose GDM), nearly a quarter of women changed diagnostic category on the second test - with a similar number going from abnormal to normal as the other way round. ${ }^{32,33}$ Reliance on a single blood glucose result in a one-step screening test, especially using lower thresholds, will have even poorer precision. Moreover, first step by GCT has the advantage that it can be performed throughout the day without the need to be in fasting conditions. ${ }^{19}$

\section{Arguments opposing two-step method}

Detractors opine that reproducibility was more of a problem with older methods of estimation of glucose, rather than with the current assays. The decision of diagnosis of GDM on the basis of two abnormal values in 100-g OGTT was also arbitrary. How GCT evolved and got incorporated into a two-step strategy is also a speculation, as it was not evidence based. ${ }^{5}$

The two-step screening preceded by GCT followed by full OGTT inevitably delays the diagnosis of GDM and therefore treatment. ${ }^{34}$ A systematic review compared the 50-g GCT and the OGTT (either 75 or $100 \mathrm{~g}$ ) to estimate the sensitivity and specificity of the GCT for GDM. ${ }^{35}$ For consecutively recruited patients, the pooled sensitivity was 0.74 for a specificity of 0.85 , meaning that the process of performing GCT and then OGTT misses around 26\% of potential GDM diagnoses. ${ }^{35}$ At certain places, the figures may be even higher, as GCT screening misses many of those with GDM with a modestly elevated fasting glucose. Recent data from the HAPO study suggested that $63 \%-73 \%$ of cases in North American centers were diagnosed on the basis of an elevated fasting glucose level alone. ${ }^{36}$ The other serious concern about using a GCT is the no-show rate for the definitive OGTT for women who are abnormal. In the Toronto Tri-Hospital Gestational Diabetes Project, 10\% of women did not proceed with the GTT; ${ }^{37}$ in a New Zealand study, the rate was $23 \% .{ }^{38}$ A recent North American report found that only $36 \%$ attended the OGTT. ${ }^{39}$ The cost-effectiveness of two-step screening has to be weighed against inadvertent delay and missed diagnosis, and with their resultant potential maternal and fetal complications. ${ }^{40}$

The result of GCT is influenced by the timing of meals, and that of testing. A recent study showed that among women with a positive GCT, those tested in the afternoon have better metabolic function and a lower risk of GDM on subsequent OGTT. ${ }^{41}$ This suggests that the time of testing may lead to misclassification of patients with markedly elevated GCT results if GDM is diagnosed without confirmation by OGTT.

A recent prospective observational study randomized 786 pregnant women to screen for GDM either with a onestep method using a 75-g OGTT using IADPSG criteria $(n=386)$ or with a two-step method with a 50-g GCT and a 100-g OGTT using the Carpenter and Coustan criteria $(n=400)$, and then analyzed the prevalence of GDM using the one- and two-step methods. This study also aimed to determine whether women diagnosed as having normal glucose tolerance by the two-step method had any worse neonatal outcome than those determined to have normal glucose tolerance by the one-step method. ${ }^{42}$ Women diagnosed with GDM by either process were treated according to the local management protocol including endocrinology review, glucose 
Table I Advantages and disadvantages of two-step and one-step testing for GDM

\begin{tabular}{lll}
\hline Characteristic & Two-step & One-step \\
\hline Method & In the two-step screening approach, a 50-g GCT followed & In the one-step screening approach, \\
& by a I00-g, 3-hour OGTT. Those who screen positive & $75-$ or 100-g OGTT is done in all patients, \\
& are followed up by an oral 100-g glucose tolerance test & without the preliminary step by GCT \\
Advantages & - Fewer false positives & - Simple to follow \\
- Avoids OGTT in more than 75\% of the women & - Easily diagnosed \\
Disadvantages & - Missed diagnosis: 75\% sensitivity with 84\% specificity as & - Poor reproducibility \\
& compared with single-step 100-g OGTT & - All women need to come in fasting state \\
& - Delay in initiating treatment even in those who test & \\
& Positive & \\
& GCT is not feasible throughout the day & \\
\hline
\end{tabular}

Abbreviations: GDM, gestational diabetes mellitus; GCT, glucose challenge test; OGTT, oral glucose tolerance test.

monitoring, dietary advice, and medication if required. The one-step method had a GDM prevalence of $14.5 \%$, and the two-step method a prevalence of $6 \%$. Women determined to have normal glucose tolerance in the two-step method had a greater risk of preeclampsia and macrosomia compared with the women defined as having normal glucose tolerance in the one-step method. On the basis of their study, authors strongly advocate elimination of the two-step process.

The advantages and disadvantages of the two-step and one-step screening protocol are summarized in Table 1.

\section{Role of $\mathrm{HbA}_{\mathrm{Ic}}$ in screening for gestational diabetes mellitus}

Although the $\mathrm{HbA}_{1 \mathrm{c}}$ reference intervals for the general population are well established, reference intervals for healthy pregnant women are not clearly defined. ${ }^{43}$ A study in India showed that by using different ranges of $\mathrm{HbA}_{1 \mathrm{c}}$ for the IADPSG criteria and the old ADA criteria, an OGTT could be avoided in approximately $40 \%$ (IADPSG) and 60\% (ADA) of women, respectively. ${ }^{44}$ However, the HAPO study showed that associations with adverse outcomes were significantly stronger with glucose measures than with $\mathrm{HbA}_{1 \mathrm{c}}{ }^{45}$

Different studies report different trends in $\mathrm{HbA}_{1 \mathrm{c}}$ during pregnancy. Increase, decrease, and no change in $\mathrm{HbA}_{1 \mathrm{c}}$ levels during pregnancy have been reported by different research groups. ${ }^{43}$ Davies and Welborn ${ }^{46}$ showed that mean $\mathrm{HbA}_{1 \mathrm{c}}$ levels tend to rise after the first trimester of pregnancy in both normal and diabetic patients. This occurs despite the fact that FBG levels tend to fall in normal pregnancy, and in the diabetic patient, control of blood glucose levels improves with the progress of pregnancy. They proposed that some factor other than the level of glucose in the blood must be operating, since if $\mathrm{HbA}_{1 \mathrm{c}}$ reflects only the average blood glucose level, it must fall with the progress of pregnancy. An explanation for this phenomenon is not clear. Pollak et $\mathrm{al}^{47}$ indicated that $\mathrm{HbA}_{1 \mathrm{c}}$ did not alter with the progress of pregnancy. Widness et $\mathrm{al}^{48}$ found in a group of 13 nondiabetic women a significant fall in $\mathrm{HbA}_{1 \mathrm{c}}$ from the first to the third trimester. McFarland et al also reported a decrease in $\mathrm{HbA}_{1 \mathrm{c}}$ from the first half of pregnancy to the third trimester. ${ }^{49}$

Altogether, the findings suggest that an $\mathrm{HbA}_{1 \mathrm{c}}$ measurement is not a useful alternative to an OGTT in pregnant women.

\section{Recommendations Adoption of IADPSG criteria}

The "IADPSG criteria" have been accepted by a variety of professional and other health care bodies including the Endocrine Society, ${ }^{13}$ Australasian Diabetes in Pregnancy Society, ${ }^{29}$ and $\mathrm{WHO},{ }^{14}$ but challenged by others including the NIH consensus panel and the ACOG. ${ }^{18,19}$ The ADA has recommended that any one of them could be used for the diagnosis of GDM. ${ }^{2}$

\section{Universal screening for overt diabetes in pregnancy}

IADPSG recommends opportunistic screening of pregnant women for preexisting diabetes at the first antenatal visit either universally in all women or in high-risk ones. The decision is being left to the discretion of the treating physician/ obstetrician. The Endocrine Society Guidelines recommend testing all pregnant women, using the same diagnostic cutoffs that are recommended in the nonpregnant population, ${ }^{13}$ whereas the ADA recommends testing women with risk factors for diabetes. ${ }^{2}$ The WHO have adopted the entire IADPSG recommendation except the adoption of $\mathrm{HbA}_{1 \mathrm{c}}$ as a diagnostic tool for diabetes in pregnancy. ${ }^{14}$ However, all women with significant risk factors must be screened for 
Table 2 High-risk factors for gestational diabetes mellitus

- Previous history of gestational diabetes mellitus

- Previously elevated blood glucose level

- Maternal age $\geq 40$ years

- Family history of diabetes mellitus (first degree relative with diabetes or a sister with gestational diabetes mellitus)

- Body mass index $>35 \mathrm{~kg} / \mathrm{m}^{2}$

- Previous macrosomia (baby with birth weight $>4,500 \mathrm{~g}$ or $>90$ th centile)

- Polycystic ovary syndrome

- Medications: corticosteroids, antipsychotics

unrecognized type 2 diabetes in early pregnancy. NIH and ACOG advocate risk factor-based screening before 24 weeks of gestation. ${ }^{18,19}$ The Australasian Diabetes in Pregnancy Society (ADIPS) recommends risk factor-based screening at the first antenatal visit. ${ }^{29}$ Women with high-risk ethnicity or a BMI of $25-35 \mathrm{~kg} / \mathrm{m}^{2}$ as their only risk factor are considered to be at "moderate risk" and should initially be screened with either a random or an FPG test. This may be followed by a 75-g OGTT (IADPSG criteria) if clinically indicated. Overweight or obese women from high-risk ethnicity should be evaluated by OGTT. OGTT is recommended at the first opportunity after conception for women having any of the high-risk factors for GDM listed in Table 2. ${ }^{49}$

\section{Two-step versus one-step testing}

The ACOG and an independent expert panel assigned by the NIH continue to promote the use of the two-step screening strategy with the nonfasting 50-g GCT and if abnormal, followed by the 3-hour 100-g OGTT using the Carpenter and Coustan criteria or the NDDG criteria. ${ }^{18,19}$ Recently, both the WHO and the Endocrine Society have advocated the use of the IADPSG criteria for the diagnosis of GDM. . $^{13,14}$ ADIPS also advocates one-step testing. ${ }^{29}$ The 2015 ADA recommendations specify that further research is needed to establish a uniform approach to diagnosing GDM, and now left the option open between the one-step IADPSG recommendation and the two-step screening strategy. ${ }^{2}$

\section{$\mathrm{HbA}_{\mathrm{Ic}}$ in screening for gestational diabetes mellitus}

$\mathrm{HbA}_{\mathrm{lc}}$ is not recommended for screening of GDM by any of the guidelines. ${ }^{2,13,14,18,19,29}$ This may be due to scarce data on this aspect. Physiological changes in red cell mass and turnover occurring during pregnancy also make interpretation of $\mathrm{HbA}_{1 \mathrm{c}}$ in relation to glycemic levels difficult in pregnancy. ${ }^{43}$ However, $\mathrm{HbA}_{1 \mathrm{c}}$ is recommended by IADPSG to rule out overt diabetes in the first trimester. ${ }^{3}$

The recommendations on various aspects of screening are summarized in Table 3.

\section{Pragmatic approach}

Screening for GDM is an essential part of obstetric care. The only controversy is how to screen for the condition. The first diagnostic criterion proposed by O'Sullivan in 1964 and its subsequent modifications (Carpenter and Coustan) were based on the maternal risk of developing type 2 diabetes, rather than on neonatal outcomes. IADPSG, in contrast, attempts to redefine GDM in terms of neonatal and short-term maternal outcomes. But we are still far from attaining a holistic criterion based on both short- and long-term outcomes. Criteria based on the maternal risk of developing type 2 diabetes would appear to underdiagnose; whereas those based on only shortterm outcomes may over diagnose GDM. A decision tree that balances neonatal outcome concern with maternal outcome and risk from treatment would seem to be the way forward, eventually. This requires more studies and data, of course.

Even though the IADPSG criteria aim to improve the perinatal outcomes, it may not be practical or feasible for every country/center to adopt it universally. As per the WHO, each health care facility needs to assess their burden of hyperglycemia in pregnancy and decide whether and how

Table 3 Recommendations on various aspects of screening

\begin{tabular}{|c|c|c|c|c|c|}
\hline $\begin{array}{l}\text { Recommending } \\
\text { body }\end{array}$ & Criteria & $\begin{array}{l}\text { Screening on first } \\
\text { antenatal visit }\end{array}$ & $\begin{array}{l}\text { Screening at 24-32 } \\
\text { weeks with OGTT }\end{array}$ & $\begin{array}{l}\text { One-step/two- } \\
\text { step screening }\end{array}$ & $\begin{array}{l}\text { HbA }_{\text {Ic }} \text { for } \\
\text { screening }\end{array}$ \\
\hline Endocrine Society, USA ${ }^{13}$ & IADPSG & Universal & Universal & One-step & No \\
\hline World Health & IADPSG & Universal or Risk & Universal & One-step & No \\
\hline Organization $^{14}$ & & factor-based & & & \\
\hline $\mathrm{ACOG}^{19}$ & Carpenter and Coustan & Risk factor-based & Universal & Two-step & No \\
\hline $\mathrm{ADA}^{2}$ & $\begin{array}{l}\text { IADPSG/Carpenter and } \\
\text { Coustan }\end{array}$ & Risk factor-based & Universal & Either of one & No \\
\hline ADIPS $^{29}$ & IADPSG & Risk factor-based & Universal & One-step & No \\
\hline
\end{tabular}

Abbreviations: ACOG, American College of Obstetricians and Gynecologists; ADA, American Diabetes Association; ADIPS, Australasian Diabetes in Pregnancy Society; IADPSG, International Association of Diabetes and Pregnancy Study Groups; OGTT, oral glucose tolerance test. 
Table 4 Pragmatic indications for one- and two-step approaches based on biopsychosocial model

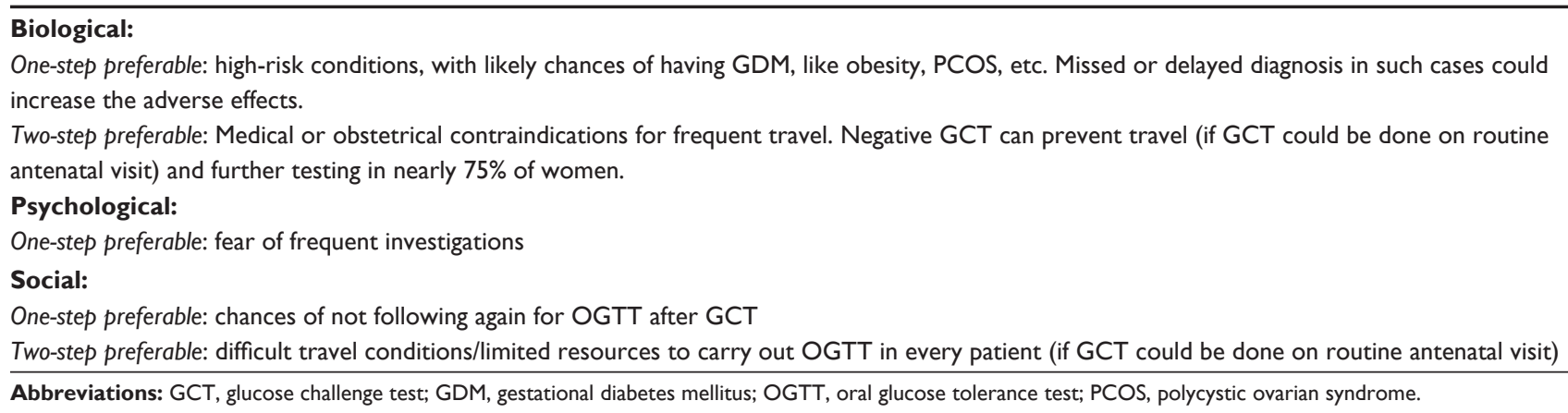

it will implement programs to test for and treat such women. ${ }^{50}$ The WHO have proposed further strategies to overcome barriers of OGTT testing with compromise on missing some cases of GDM..$^{50}$ But again, the suggestions are made on the basis of limited studies, and the applicability of the results of one study needs to be evaluated in the indigenous population before a particular strategy is adopted. While developed health care practices may follow a two-step method, a onestep approach may be more useful for situations where access or travel to health care centers is limited or arduous. The benefits of a second test, performed in a second visit, must be balanced against the cost and inconvenience of travel. Travel is also associated with health risks. However, if GCT is accomplished on routine antenatal visit, then a two-step strategy may be beneficial in such cases. ${ }^{5}$ The universal screening to rule out overt diabetes should, however, be adopted in ethnicities/countries with a high prevalence of diabetes. ${ }^{13} \mathrm{We}$ summarize the pragmatic indications for the one- and twostep approaches on the basis of a biopsychosocial model in Table 4 and an overall pragmatic approach based on guidance from various professional bodies in Figure 1.

\section{Future directions}

It is felt that most guidelines fail to address the need and constraints of low-resource settings, where the demand for clear and simple directions is the greatest. The screening protocols are often based on evidence from scientific research carried out by well-resourced academic institutions. However, the typical clinical settings in low- and middle-income countries with poor resources and high disease burden face conditions that are often far away from these ideal settings. This calls into question the feasibility of applying screening procedures and diagnostic criteria. Future studies need to address these issues in great detail. ${ }^{50}$ Data on the preference of pregnant women for a particular diagnostic test are unavailable. Future studies should also evaluate the impact of any strategy on personal satisfaction, quality of life, or psychological aspects of individual patients. Patient preferences, convenience of testing versus outcome of testing and treatment should also be taken into account. This is indeed an important future research imperative as without patient compliance, evidence-based screening decisions are useless. As per our clinical experience and that of other experts, pregnant women are more concerned about the outcome of their pregnancy than by the relatively minor inconveniences of diagnostic testing, GDM labeling and possible treatment of limited duration. ${ }^{14}$

The NIH consensus panel identifies as a priority the conduct of a new RCT evaluating outcomes in women currently classified as "normal" according to prevalent US criteria but who would be considered abnormal by the IADPSG and the ADA. ${ }^{18}$ The diagnosis of GDM is generally made in the late second or early third trimester. Early detection and treatment may potentially improve outcomes. However, there is a dearth of evidence in this area. Well-designed studies are needed to determine the most appropriate means of testing for GDM in early pregnancy and to explore the outcomes of early treatment interventions.

The adoption of IADPSG criteria by some professional organizations is mainly due to potential benefits (to mother and child) in the prevention of short-term pregnancies and perinatal outcomes. Data on potential long-term benefits to the health of the mother and her offspring are still scarce. Future studies and/or follow-up of previous cohorts like the HAPO study could answer this question. ${ }^{14}$

\section{Summary}

Apart from the debate on other issues, the first step should be the implementation of universal screening to identify more severe cases of GDM. This step will already be a major one in most countries. The different viewpoints from experts and guidelines underscore the fact that there are data to support each strategy (IADPSG vs Carpenter and Coustan criteria; one-step 


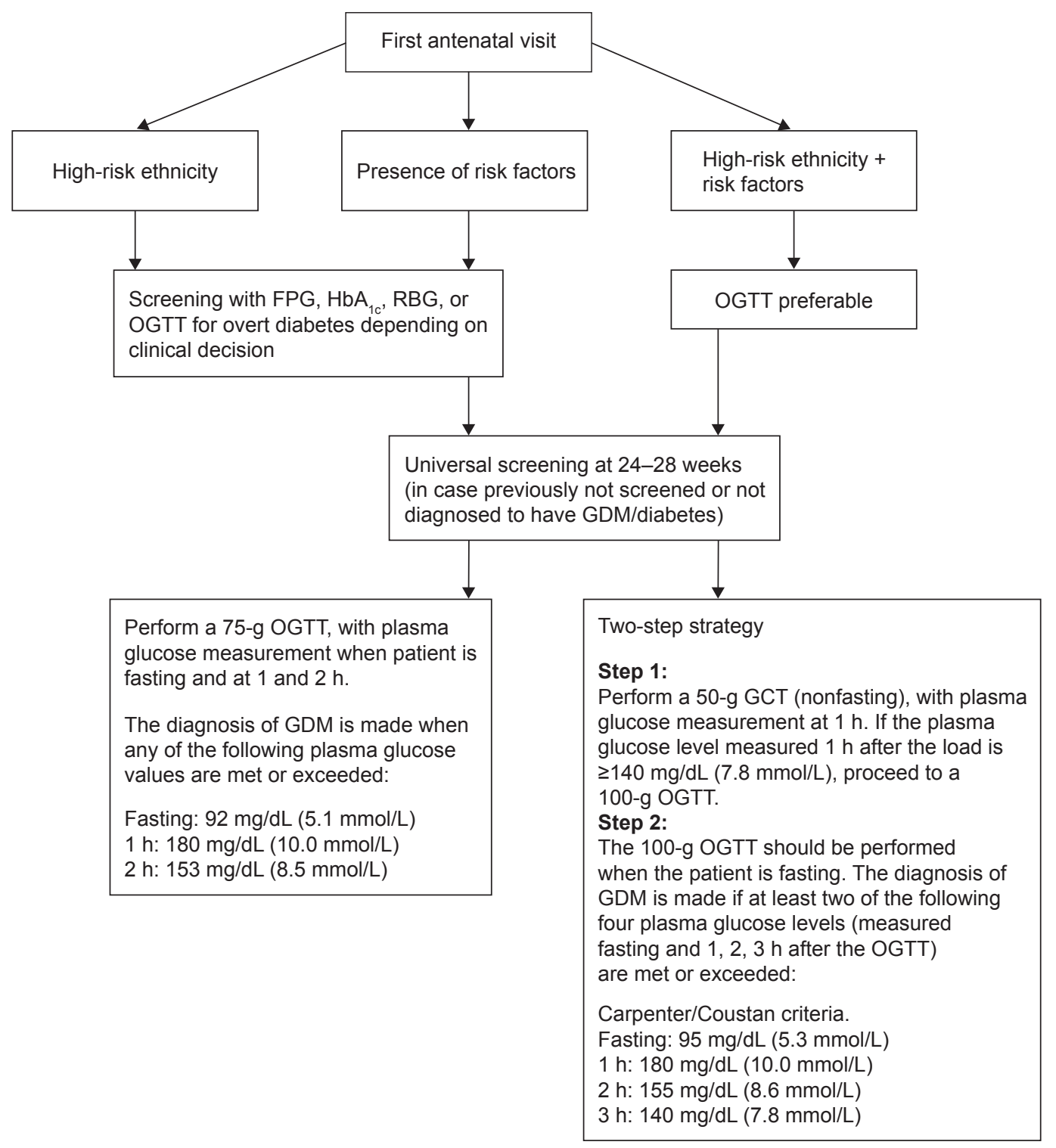

Figure I Pragmatic approach to screen women for overt diabetes and GDM.

Abbreviations: FPG, fasting plasma glucose; RBG, random blood glucose; OGTT, oral glucose tolerance test; GDM, gestational diabetes mellitus; GCT, glucose challenge test; h, hour.

vs two-step screening). The decision on which strategy to implement must therefore be made on the basis of the relative role of cost considerations, and availability of infrastructure locally, nationally, and internationally. In addition, patient preferences should be taken into account as part of any screening strategy.

\section{Disclosure}

The authors report no conflicts of interest in this work.

\section{References}

1. American Diabetic Association. Diagnosis and classification of diabetes mellitus. Diabetes Care. 2006;29(Suppl 1):S43-S48.

2. American Diabetic Association. Classification and diagnosis of diabetes mellitus. Diabetes Care. 2015;38(Suppl 1):S8-S16.
3. International Association of Diabetes and Pregnancy Study Groups Consensus Panel, Metzger BE, Gabbe SG, Persson B, et al. International association of diabetes and pregnancy study groups recommendations on the diagnosis and classification of hyperglycemia in pregnancy. Diabetes Care. 2010;33(3):676-682.

4. Buchanan TA, Xiang AH, Page KA. Gestational diabetes mellitus: risks and management during and after pregnancy. Nat Rev Endocrinol. 2012;8(11):639-649.

5. McIntyre HD, Colagiuri S, Roglic G, Hod M. Diagnosis of GDM: a suggested consensus. Best Pract Res Clin Obstet Gynaecol. 2015;29(2): 194-205. doi:10.1016/j.bpobgyn.2014.04.022.

6. Houshmand A, Jensen DM, Mathiesen ER, Damm P. Evolution of diagnostic criteria for gestational diabetes mellitus. Acta Obstet Gynecol Scand. 2013;92(7):739-745.

7. Coustan DR. Diagnosis of gestational diabetes. Scand J Clin Lab Invest Suppl. 2014;244:27-33.

8. O'Sullivan JB, Mahan CM. Criteria for the oral glucose tolerance test in pregnancy. Diabetes. 1964;13:278-285. 
9. National Diabetes Data Group. Classification and diagnosis of diabetes mellitus and other categories of glucose intolerance. Diabetes. 1979; 28(12):1039-1057.

10. Carpenter MW, Coustan DR. Criteria for screening tests for gestational diabetes. Am J Obstet Gynecol. 1982;144(7):768-773.

11. World Health Organization. Definition, Diagnosis and Classification of Diabetes Mellitus and its Complications: Report of a WHO Consultation. Part 1: Diagnosis and Classification of Diabetes Mellitus. WHO/ NCD/NCS/99. 2nd ed. Geneva, Switzerland: World Health Organization; 1999.

12. HAPO Study Cooperative Research Group, Metzger BE, Lowe LP, Dyer AR, et al. Hyperglycemia and adverse pregnancy outcomes. N Engl J Med. 2008;358(19):1991-2002.

13. Blumer I, Hadar E, Hadden DR, et al. Diabetes and pregnancy: an endocrine society clinical practice guideline. J Clin Endocrinol Metab. 2013;98(11):4227-4249.

14. World Health Organization. Diagnostic criteria and classification of hyperglycaemia first detected in pregnancy. Diabetes Res Clin Pract. 2014;103(3):341-363.

15. McIntyre HD, Metzger BE, Coustan DR, et al. Counterpoint: establishing consensus in the diagnosis of GDM following the HAPO study. Curr Diab Rep. 2014;14(6):497.

16. Long H, Cundy T. Establishing consensus in the diagnosis of gestational diabetes following HAPO: where do we stand? Curr Diab Rep. 2013; 13(1):43-50.

17. Ryan EA. Diagnosing gestational diabetes. Diabetologia. 2011;54(3): 480-486.

18. National Institutes of Health Consensus Development Conference Panel. National Institutes of Health consensus development conference statement: diagnosing gestational diabetes mellitus, March 4-6, 2013. Obstet Gynecol. 2013;122(2 Pt 1):358-369.

19. Committee on Practice Bulletins - Obstetrics. Practice Bulletin No 137: gestational diabetes mellitus. Obstet Gynecol. 2013;122(2 Pt 1): 406-416

20. Long H. Diagnosing gestational diabetes: can expert opinions replace scientific evidence? Diabetologia. 2011;54(9):2211-2213.

21. Crowther CA, Hiller JE, Moss JR, McPhee AJ, Jeffries WS, Robinson JS. Effect of treatment of gestational diabetes mellitus on pregnancy outcomes. N Engl J Med. 2005;352(24):2477-2486.

22. Landon MB, Spong CY, Thom E, et al. A multicenter, randomized trial of treatment for mild gestational diabetes. N Engl J Med. 2009;361(14): 1339-1348.

23. Horvath K, Koch K, Jeitler K, et al. Effects of treatment in women with gestational diabetes mellitus: systemic review and meta-analysis. $B M J$. 2010;340:c1395.

24. McIntyre HD. Diagnosing gestational diabetes mellitus: rationed or rationally related to risk? Diabetes Care. 2013;36(10):2879-2880.

25. Catalano PM, McIntyre HD, Cruickshank JK, et al. The Hyperglycemia and Adverse Pregnancy Outcome Study: associations of GDM and obesity with pregnancy outcomes. Diabetes Care. 2012;35(4):780-786.

26. HAPO Study Cooperative Research Group. Hyperglycaemia and Adverse Pregnancy Outcome (HAPO) Study: associations with maternal body mass index. BJOG. 2010;117(5):575-584

27. International Association of Diabetes and Pregnancy Study Groups (IADPSG) Consensus Panel Writing Group and the Hyperglycemia and Adverse Pregnancy Outcome (HAPO) Study Steering Committee, Metzger BE, Gabbe SG, Persson B, et al. The diagnosis of gestational diabetes mellitus: new paradigms or status quo? J Matern Fetal Neonatal Med. 2012;25(12):2564-2569.

28. Falavigna M, Schmidt MI, Trujillo J, et al. Effectiveness of gestational diabetes treatment: a systematic review with quality of evidence assessment. Diabetes Res Clin Pract. 2012;98(3):396e405.

29. Australasian Diabetes In Pregnancy Society (ADIPS) Consensus Guidelines for the Testing and Diagnosis of Gestational Diabetes Mellitus in Australia. Available from: http://www.bhs.org.au/airapps/Services/ au/org/bhs/govdoc/files/references/14440.pdf. Accessed January 27, 2015 .
30. Donovan L, Hartling L, Muise M, Guthrie A, Vandermeer B, Dryden DM. Screening tests for gestational diabetes: a systematic review for the US Preventive Services Task Force. Ann Intern Med. 2013;159(2): $115-122$.

31. Zhu WW, Yang HX, Wei YM, et al. Evaluation of the value of fasting plasma glucose in the first prenatal visit to diagnose gestational diabetes mellitus in china. Diabetes Care. 2013;36(3):586-590.

32. Harlass FE, Brady K, Read JA. Reproducibility of the oral glucose tolerance test in pregnancy. Am J Obstet Gynecol. 1991;164(2):564-568.

33. Catalano PM, Drago NM, Amini SB. Reproducibility of the oral glucose tolerance test in pregnant women. Am J Obstet Gynecol. 1993; 169(4):874-881

34. Moses RG, Cheung NW. Point: universal screening for gestational diabetes mellitus. Diabetes Care. 2009;32(7):1349-1351.

35. van Leeuwen M, Louwerse MD, Opmeer BC, et al. Glucose challenge test for detecting gestational diabetes mellitus: a systematic review. BJOG. 2012;119(4):393e401.

36. Sacks DA, Hadden DR, Maresh M, et al. Frequency of gestational diabetes mellitus based on IADPSG consensus panel - recommended criteria at collaborating centers. Diabetes Care. 2012;35(3):526-528.

37. Sermer M, Naylor CD, Gare DJ, et al. Impact of increasing carbohydrate intolerance on maternal-fetal outcomes in 3637 women without gestational diabetes. The Toronto Tri-Hospital Gestational Diabetes Project. Am J Obstet Gynecol. 1995;173(1):146-156.

38. Yapa M, Simmons D. Screening for gestational diabetes mellitus in a multiethnic population in New Zealand. Diabetes Res Clin Pract. 2000; 48(3):217-223.

39. Sievenpiper JL, McDonald SD, Grey V, Don-Wauchope AC. Missed follow-up opportunities using a two-step screening approach for gestational diabetes. Diabetes Res Clin Pract. 2012;96(2):e43-e46.

40. Simmons D, Moses RG. Gestational diabetes mellitus: to screen or not to screen? Is this really still a question? Diabetes Care. 2013;36(10): 2877-2878.

41. Goldberg RJ, Ye C, Sermer M, et al. Circadian variation in the response to the glucose challenge test in pregnancy: implications for screening for gestational diabetes mellitus. Diabetes Care. 2012;35(7): $1578-1584$.

42. Sevket O, Ates S, Uysal O, Molla T, Dansuk R, Kelekci S. To evaluate the prevalence and clinical outcomes using a one-step method versus a two-step method to screen gestational diabetes mellitus. J Matern Fetal Neonatal Med. 2014;27(1):36-41.

43. Rafat D, Ahmad J. HbA ${ }_{1 \mathrm{c}}$ in pregnancy. Diabetes Metab Syndr. 2012; 6(1):59-64.

44. Raiput R, Yadav Y, Raiput M, et al. Utility of $\mathrm{HbA}_{1 \mathrm{c}}$ for diagnosis of gestational diabetes mellitus. Diabetes Res Clin Pract. 2012;98(1): 104-107.

45. Lowe P, Metzger BE, Dyer AR, et al. Hyperglycemia and Adverse Pregnancy Outcome (HAPO) Study: associations of maternal A1C and glucose with pregnancy outcomes. Diabetes Care. 2012;35(3): 574-580.

46. Davies DM, Welborn TA. Glycosylated haemoglobin in pregnancy. Aust N Z J Obstet Gynaecol. 1980;20(3):147-150.

47. Pollak A, Widness JA, Schwartz R. Minor hemoglobins: an alternative approach for evaluating glucose control in pregnancy. Biol Neonate. 1979;36(3-4):185-192.

48. Widness JA, Schwartz HC, Kahn CB, Oh W, Schwartz R. Glycohemoglobin in diabetic pregnancy: a sequential study. Am J Obstet Gynecol. 1980;136(8):1024-1029.

49. McFarland KF, Catalano EW, Keil JE, McFarland DE. Glycosylated haemoglobin in diabetic and nondiabetic pregnancies. South Med J. 1981;74(4):410-412.

50. Colagiuri S, Falavigna M, Agarwal MM, et al. Strategies for implementing the WHO diagnostic criteria and classification of hyperglycaemia first detected in pregnancy. Diabetes Res Clin Pract. 2014;103(3): 364-372. 
International Journal of Women's Health

Dovepress

\section{Publish your work in this journal}

The International Journal of Women's Health is an international, peerreviewed open-access journal publishing original research, reports, editorials, reviews and commentaries on all aspects of women's healthcare including gynecology, obstetrics, and breast cancer. The manuscript management system is completely online and includes Visit http://www.dovepress.com/testimonials.php to read real quotes from published authors.

Submit your manuscript here: http://www.dovepress.com/international-journal-of-womens-health-journal 